\title{
Perfil sociodemográfico e ocupacional de trabalhadores da Prefeitura de um Campus Universitário
}

\author{
Sociodemographic and occupational profile of Campus Hall workers \\ from a public university
}

\author{
Aline Loiola Moura ${ }^{1}$; Maria do Carmo Lourenço Haddad²; Marli Terezinha Oliveira \\ Vannuchi $^{3}$; Larissa Gutierrez de Carvalho Silva ${ }^{4}$; Mariana Ângela Rossaneis ${ }^{5}$
}

\section{Resumo}

O estudo objetivou identificar o perfil sociodemográfico e ocupacional de trabalhadores da prefeitura de um campus universitário público. Trata-se de uma pesquisa quantitativa, descritiva, sendo utilizado um formulário constituído por dados sociodemográficos e ocupacionais. A amostra foi composta por 307 trabalhadores. Os resultados demonstraram que a maior parte dos funcionários era do sexo masculino (85,3\%), em união estável (73,3\%), na faixa etária de 41 a 60 anos (78,2\%), sendo evidenciado que o envelhecimento da força de trabalho é marcante. Em relação aos dados ocupacionais, a maior parcela de trabalhadores atuava no cargo de auxiliar operacional (71,9\%), com tempo de admissão na instituição superior a 11 anos $(79,8 \%)$, realizavam horas extras $(75,5 \%)$, sendo que $5,5 \%$ dos servidores eram readaptados ou readequados. Conclui-se que é relevante identificar as características dos trabalhadores para implementar intervenções institucionais com o intuito de proporcionar condições que favoreçam a prevenção de patologias relacionadas às atividades laborais e garantir aos trabalhadores a manutenção de sua capacidade funcional.

Palavras-chave: Inquéritos epidemiológicos. Saúde do trabalhador. Perfil de saúde. Enfermagem. Envelhecimento.

\begin{abstract}
The study aimed to identify the sociodemographic and occupational profile of workers in the campus hall of a university. It is a quantitative, descriptive research, being used a form consisting of demographic and occupational data. The sample consisted of 307 workers. The results showed that most employees were male $(85.3 \%)$, in a stable relationship $(73.3 \%)$, aged $41-60$ years $(78.2 \%)$, and demonstrated that aging workforce is striking. Regarding occupational data, the largest share of workers worked in the position of operating aid $(71.9 \%)$, with admission time in the institution greater than 11 years $(79.8 \%)$, performed overtime $(75.5 \%)$, and $5.5 \%$ of the workers were readjusted or readequated. We conclude that it is important to identify the characteristics of workers to implement institutional interventions aimed at providing conditions that favor the prevention of diseases related to work activities and ensuring that workers maintain their functional capacity.
\end{abstract}

Keywords: Health surveys. Occupational health. Health profile. Nursing. Aging.

\footnotetext{
${ }^{1}$ Mestre em Enfermagem/Universidade Estadual de Londrina, Brasil.

2 Doutora em Enfermagem/ Escola de Enfermagem de Ribeirão Preto/Universidade de São Paulo, Brasil.

${ }^{3}$ Doutora em Saúde Pública/ Universidade de São Paulo, Brasil.

${ }^{4}$ Doutoranda em Enfermagem/Escola de Enfermagem de Ribeirão Preto/Universidade de São Paulo, Brasil.

${ }^{5}$ Doutoranda em Enfermagem/Universidade Estadual de Maringá, Brasil.
} 


\section{Introdução}

O processo de trabalho deve ser compreendido como o modo pelo qual o homem produz e reproduz a sua própria existência. A concepção histórica, materialista e lógica procura apontar que cada geração transmite uma massa de forças produtivas, de capitais e de circunstâncias, que é, por um lado, muito modificada pela nova geração, mas, por outro lado, lhe dita suas condições de existência e lhe imprime um desenvolvimento determinado, um caráter específico (RIBEIRO et al., 2012).

As instituições empregadoras devem reconhecer os servidores como o seu maior e mais precioso patrimônio, considerando que esses profissionais possuem valores, motivações e necessidades de alcançarem sucesso com dignidade e competência. Devem ainda deixar de serem tratados somente como recursos, passando a serem vistos como verdadeiros meios, com o intuito de atingir os objetivos da organização (PIRES et al., 2013).

Atualmente, o Brasil está passando por um processo de transição demográfica, marcado pelo envelhecimento da população, tanto em idade produtiva como também da força de trabalho, tornando-se indispensável à indagação pela qualidade de vida das pessoas no processo funcional (FELLI; TRONCHIN, 2010).

A qualidade de vida no trabalho reflete diretamente na vida social, e muitas vezes no relacionamento familiar do trabalhador. Contudo, a qualidade dos serviços prestados também é afetada pelas más condições de trabalho, devido ao estresse, ao cansaço e à fadiga provocada por um ambiente de trabalho inadequado (ANDRADE; VEIGA, 2012).

$\mathrm{Na}$ busca pela manutenção da capacidade laboral, várias instituições têm evidenciado um real interesse na prevenção de acidentes, doenças ocupacionais, perda da capacidade funcional, visando uma qualidade de vida no trabalho, através de atividades isoladas ou por vezes em parcerias. Estas instituições, através de suas competências, buscam aumentar a eficácia de suas ações e desenvolver alternativas para lidar com esta importante questão social, com um enfoque importante para os trabalhadores mais experientes (LIMA, 2009).

A Organização Mundial de Saúde (OMS) definiu como trabalhador em fase de envelhecimento aquele com 45 anos de idade ou mais. Assume-se que a partir dessa idade as perdas de algumas capacidades funcionais tendem a se acentuar caso medidas preventivas não sejam adotadas, bem como se as condições de trabalho não forem adequadas. Condições inadequadas de trabalho podem ser traduzidas em riscos de natureza física, química, biológica ou organizacional, que podem resultar no afastamento precoce do indivíduo do mercado de trabalho (CAMARANO; PASINATO, 2008).

Assim, vale salientar a necessidade da adoção de medidas e de políticas adequadas às condições de trabalho para a população que envelhece, de forma a manter sua satisfação e capacidade funcional, e adiar sua saída das atividades laborais, buscando o equilíbrio entre as novas pressões da economia e a busca de um ambiente organizacional saudável e prazeroso (FRANÇA; SOARES, 2009).

Dentre os diversos setores de uma universidade pública, a Prefeitura é um órgão representativo e tem como responsabilidade o planejamento, elaboração, execução e controle de projetos de estrutura física do campus (UNIVERSIDADE ESTADUAL DE LONDRINA, 2014a). Considerando que os trabalhadores lotados neste órgão são responsáveis pela manutenção da estrutura física e funcionamento de vários setores da instituição, o que exige esforço físico, bem como é importante verificar as características desta força de trabalho e identificar fatores que possam interferir na diminuição da capacidade 
laboral e envelhecimento funcional destes trabalhadores, possibilitando uma intervenção efetiva da equipe de saúde multidisciplinar, além de que não foram desenvolvidas pesquisas no sentido de verificar as características desta força de trabalho, o presente estudo teve como objetivo caracterizar o perfil sociodemográfico e ocupacional de trabalhadores da prefeitura de um campus universitário público.

\section{Metodologia}

Trata-se de um estudo de abordagem quantitativa, do tipo descritivo, transversal, enfatizando o perfil sociodemográfico e ocupacional de trabalhadores da prefeitura de um campus universitário público, que é constituído em sua totalidade por mais de 22.000 pessoas, assim distribuídas: 5.220 servidores, que são representados por docentes e técnicos, 13.125 graduandos e 4.418 pósgraduandos (UNIVERSIDADE ESTADUAL DE LONDRINA, 2014b).

No período da pesquisa, a prefeitura contava com 404 servidores e todos foram convidados a participar do estudo, independente de idade, sexo e categoria profissional. Os funcionários que estavam em férias, licença prêmio, licença médica ou que não aceitaram participar da pesquisa não foram incluídos na coleta de dados.

A coleta de dados foi realizada no período de março a junho de 2010 por meio de entrevistas com auxílio de um formulário constituído por dados sociodemográficos e ocupacionais (DOMANSKY, 2004). Para tanto, foram recrutadas e capacitadas seis acadêmicas de graduação do curso de enfermagem da UEL, sendo realizados três encontros com o intuito de esclarecer os objetivos do estudo, a composição e estrutura dos instrumentos e as etapas da pesquisa.
Os trabalhadores foram abordados individualmente, nos respectivos locais de trabalho, durante o expediente de serviço. Para tanto, os entrevistadores tiveram em mãos a lista nominal de todos os trabalhadores por setor e turno de trabalho.

O perfil sociodemográfico foi identificado por meio das variáveis: idade, sexo, cor da pele, situação conjugal e escolaridade. O perfil ocupacional foi traçado pela caracterização da ocupação atual, tempo de exercício profissional na instituição, função exercida, readequação ou readaptação funcional, turno de trabalho, posição adotada no trabalho, carga horária diária e semanal, tempo de trabalho na função, realização de horas extras, outro vínculo empregatício e desenvolvimento de atividades escolares ou acadêmicas.

Os dados coletados foram digitados e armazenados em banco de dados no formato Epi Info versão 3.5.1 para Windows ${ }^{\circledR}$. Foi utilizada estatística descritiva, adotando-se distribuições e frequências (absoluta e relativa), medidas descritivas (média, mediana, percentis, variação mínima e máxima).

O presente estudo obteve aprovação do Comitê de Ética em Pesquisa da UEL, sob o parecer $n^{0}$ 147/2009. Todos os entrevistados foram orientados sobre a pesquisa e assinaram o Termo de Consentimento Livre e Esclarecido (TCLE).

\section{Resultados}

A população do estudo foi composta por 307 trabalhadores da prefeitura do campus, sendo que as perdas foram justificadas como: 15 estavam em férias, 7 em licença prêmio, 12 em licença médica e 63 não aceitaram participar da pesquisa. A tabela 1 demonstra as principais características sóciodemográficas dos entrevistados. 
Tabela 1 - Distribuição das características sóciodemográficas dos trabalhadores da prefeitura de um campus universitário público. Londrina - PR, 2010.

\begin{tabular}{lrr} 
Característica & N & \% \\
Sexo & 45 & 14,7 \\
Feminino & 262 & 85,3 \\
Masculino & 21 & 6,8 \\
Idade (anos) & 46 & 15,0 \\
$<30$ & 104 & 33,9 \\
$30-40$ & 100 & 32,6 \\
$41-50$ & 36 & 11,7 \\
$51-60$ & & \\
$>60$ & 40 & 13,0 \\
Situação conjugal & 225 & 73,3 \\
Solteiro & 33 & 10,8 \\
União estável & 9 & 2,9 \\
Separado/divorciado & & \\
Viúvo & 189 & 61,6 \\
Cor da pele & 118 & 38,4 \\
Branca & & \\
Não branca & 293 & 95,4 \\
Religião & 14 & 4,6 \\
Sim & & 21,8 \\
Não & 67 & 51,1 \\
Escolaridade & 157 & 21,5 \\
Fundamental & 66 & 5,6 \\
Médio & 17 & \\
Superior & & \\
Pós graduação & & \\
\hline
\end{tabular}

Fonte: Os autores.

Verificou-se a prevalência do sexo masculino, com $85,3 \%$, sendo que a faixa etária variou de 20 a 69 anos. A média das idades foi de 48 anos (Desvio Padrão = 10 anos), o que determina um coeficiente de variação de $21 \%$.

Quanto à situação conjugal 73,3\% dos entrevistados relataram ter uma união estável, 13,0\% eram solteiros. Em relação à cor da pele, a maioria $(61,6 \%)$ referiu ter pele branca. Comprovou-se a alta adesão dos entrevistados a práticas religiosas, visto que $95,4 \%$ dos trabalhadores declararam possuir uma religião.

Em relação à escolaridade, verificou-se que 51,1\% dos entrevistados completaram o ensino médio, no entanto $13,7 \%$ não possuíam o nível fundamental. Verifica-se ainda a presença de trabalhadores com especialização latu sensu e stricto senso (5,6\%).

A Tabela 2 apresenta as características ocupacionais dos trabalhadores entrevistados. Quanto à área de ocupação auto referida por trabalhadores da instituição em estudo, observou-se que $71,9 \%$ exercem função de auxiliar operacional, 14,9\% ocupam cargos técnicos e $13 \%$ ocupam cargos administrativos. Devido à diversidade de ocupação verificada entre os trabalhadores, as mesmas foram agrupadas de acordo com sua função laborativa, independente de seus subsetores de origem.

Grande parte da população estudada $(79,8 \%)$ possui tempo de admissão superior a 11 anos, sendo que $27 \%$ trabalham na universidade há pelo menos 21 anos. O turno prevalente foi o período integral $(84,4 \%)$ e a posição mais evidente adotada no trabalho foi variável $(49,2 \%)$, alternando entre ficar sentado, em pé e caminhar.

Houve um relato importante de realização de horas extras (75,5\% dos entrevistados), o que justifica um único vínculo empregatício em 93,5\% dos trabalhadores. A maioria (71,3\%) não estava estudando no momento da pesquisa.

Destaca-se ainda a presença de readaptados ou readequados, representando $5,5 \%$ dos trabalhadores. 
Tabela 2 - Distribuição das características ocupacionais dos trabalhadores da prefeitura de um campus universitário público. Londrina - PR, 2010.

\begin{tabular}{|c|c|c|}
\hline CARACTERÍSTICAS OCUPACIONAIS & $\mathbf{N}$ & $\%$ \\
\hline \multicolumn{3}{|l|}{ Área de atuação profissional } \\
\hline Auxiliar operacional* & 221 & 71,9 \\
\hline Técnico** & 46 & 14,9 \\
\hline Administrativo*** & 40 & 13,0 \\
\hline \multicolumn{3}{|l|}{ Tempo de serviço (em anos) } \\
\hline 5 ou menos & 45 & 14,7 \\
\hline $6-10$ & 17 & 5,5 \\
\hline $11-15$ & 81 & 26,4 \\
\hline $16-20$ & 81 & 26,4 \\
\hline 21 ou mais & 83 & 27 \\
\hline \multicolumn{3}{|l|}{ Turno } \\
\hline Manhã & 4 & 1,3 \\
\hline Tarde & 5 & 1,6 \\
\hline Noite & 16 & 5,2 \\
\hline Integral & 259 & 84,4 \\
\hline Alternado & 20 & 6,5 \\
\hline Outro & 3 & 1,0 \\
\hline \multicolumn{3}{|l|}{ Posição adota no trabalho } \\
\hline Sentada & 62 & 20,2 \\
\hline Em pé & 60 & 19,5 \\
\hline Caminhando & 30 & 9,8 \\
\hline Alternância & 151 & 49,2 \\
\hline Outra & 4 & 1,3 \\
\hline \multicolumn{3}{|l|}{ Realiza hora extra (HE) } \\
\hline Sim & 233 & 75,9 \\
\hline Não & 74 & 24,1 \\
\hline \multicolumn{3}{|l|}{$\begin{array}{l}\text { Nao } \\
\text { Outro vínculo empregatício }\end{array}$} \\
\hline Sim & 20 & 6,5 \\
\hline Não & 287 & 93,5 \\
\hline \multicolumn{3}{|l|}{ Realiza atividades escolares ou acadêmicas } \\
\hline Sim & 88 & 28,7 \\
\hline Não & 219 & 71,3 \\
\hline \multicolumn{3}{|l|}{ Readaptado/readequado } \\
\hline Sim & 17 & 5,5 \\
\hline Não & 290 & 94,5 \\
\hline
\end{tabular}

* Auxiliar operacional: motorista, segurança, zelador, jardineiro, auxiliar de manutenção.

** Técnico: eletricista, técnico de manutenção, técnico em assuntos universitários.

*** Técnico Administrativo: telefonista, auxiliar administrativo, contador, encarregado de seção.

Fonte: Os autores.

\section{Discussão}

Considerando que prevalece o sexo masculino na população deste estudo, destaca-se que ainda é marcante em algumas instituições, a divisão sexual do trabalho. Estudo mostra que para a manutenção da família, através das épocas, sempre atribuiu ao homem a função de principal provedor financeiro das necessidades da casa, e para a mulher permanece a função de reprodução biológica da força de trabalho, ou seja, a procriação e criação dos filhos (BRUSCHINI; RICOLDI, 2009).

Cabe ressaltar também que as atividades laborais no setor pesquisado tem uma alta exigência física, o que pode justificar o quantitativo elevado de trabalhadores do sexo masculino. Essa vinculação de determinada tarefa ou atividade ao gênero possui uma importante determinação cultural e social, que define as áreas de atuação do homem e da mulher a partir de critérios mais ou menos rígidos. Apesar da persistência desta discriminação, habilidades femininas e masculinas começam a ser consideradas de maneira mais equitativa (SANTOS, 2006).

Em relação à idade, evidenciou-se que mais da metade da população está na faixa etária entre 41 a 60 anos, mostrando o envelhecimento da força de trabalho. Estudo revela que o fator idade é risco adicional para o desenvolvimento de problemas de saúde levando o trabalhador ao 
envelhecimento precoce, que impedem a pessoa de continuar executando determinada tarefa, ou até mesmo manter-se no seu trabalho, que é uma das preocupações do sistema previdenciário mundial, pois a quantidade de pessoas idosas está aumentando, enquanto o número de pessoas que irão repor esta força de trabalho não está crescendo na mesma proporção (FISCHER, 2014). Um trabalhador com a capacidade funcional diminuída torna-se menos produtivo, tem mais dificuldades nas tomadas de decisões e conseqüentemente torna-se mais ausente no trabalho (MARTINEZ; LATORRE; FISCHER, 2010).

Em relação à situação conjugal, o estudo mostrou que a maioria possuía união estável. Os impactos da família sobre o trabalho indicam que relações familiares conturbadas e estresse relacionado à vida conjugal tendem a se relacionar positivamente com absenteísmo e negativamente com desempenho no trabalho (SADIR; BIGNOTTO, 2010).

Considerando que parcela significativa desta população possui no mínimo ensino médio completo, pode-se afirmar que este é um fator facilitador para as ações de educação em saúde e fator protetor para déficits cognitivos (PASKULINL; VIANNALL, 2007), de modo a facilitar para o individuo a busca por hábitos de vida mais saudáveis.

Destaca-se a significativa proporção de trabalhadores que ocupam cargos de auxiliares operacionais (motoristas, seguranças, zeladores, jardineiros, auxiliares de manutenção), que, por desenvolverem atividades que requerem esforço físico por meio de levantamento de peso, longas jornadas em pé e exposição ao calor e frio, podem apresentar problemas de saúde relacionados ao trabalho, como doenças osteomusculares, dorsocervicolombalgias, estresse, danos ao sistema nervoso, o que tende a diminuir consideravelmente a capacidade para o trabalho destes indivíduos (MARTINEZ; LATORRE; FISCHER, 2010).

Verificou-se que $85,3 \%$ dos funcionários têm mais de 10 anos de trabalho na instituição, sendo que destes, 72,5\% trabalham todos estes anos na mesma função, o que pode levar ao desgaste físico e mental associado a execução contínua das mesmas atividades por um longo período. Esta situação pode ser agravada pela ocorrência de acidentes e doenças ocupacionais, sendo que o Brasil ainda ocupa uma posição critica em relação a estes indicadores quando comparado a nações mais desenvolvidas. Portanto, é preciso considerar que o homem passa pelo menos um terço do dia em jornadas de trabalho, durante muitos anos de sua vida exercendo atividades repetitivas, e, portanto, o trabalho é um condicionante da qualidade de vida e estado de saúde do homem (COSTA et al., 2013).

Apesar de pequena parcela de a população trabalhar no período noturno $(5,2 \%)$, vale ressaltar que realizar atividades laborais em horários não diurnos pode levar os trabalhadores a ter pior desempenho de seus afazeres, a expô-los a maiores riscos de acidentes de trabalho e, de forma mais acentuada, a estressores ambientais, que podem levá-los à incapacidade e envelhecimento funcional precoce (NEVES et al., 2010).

Em relação a outro vínculo empregatício 6,5\% dos entrevistados, afirmou ter outro emprego concomitante, fato que pode comprometer a saúde destes trabalhadores, expondo-o a maiores riscos no ambiente de trabalho devido ao cansaço, desgaste físico e sobrecarga laboral. Dos funcionários que faziam hora-extra, 83,3\% trabalhavam em turno integral e $9 \%$ trabalhavam no período da manhã. $\mathrm{O}$ fato dos funcionários necessitarem de aumentar seu poder aquisitivo os leva a busca por hora-extra e duplicidade de vínculos empregatícios, o que também pode prejudicar sua capacidade funcional.

Em relação à posição adotada no trabalho, a literatura aponta que posturas desfavoráveis ocasionam aumento de fadiga no trabalhador e levam ao longo do tempo a lesões graves. Levando em consideração o mencionado, as principais conseqüências de determinadas posturas habituais são: na postura de pé prolongada podem ocorrer 
congestão das pernas, formação de edemas ou varizes e deformação dos pés; para a postura sentada curvado, há compressão dos órgãos internos e prováveis distúrbios digestivos; já na postura curvada (de pé, sentado, ajoelhado) podem ser identificados desvios da coluna vertebral, afecções e lesões dos discos intervertebrais (QUEMELO et al., 2009).

Quanto à presença de trabalhadores readaptados e readequados na população deste estudo, ressaltase que o fato destes trabalhadores exercerem atividades com importante demanda física associado ao envelhecimento cronológico e funcional pode ter influenciado na solicitação desta medida administrativa. A readaptação/readequação é um termo utilizado para encaminhar a situação de trabalhadores que procuram assistência médica e são considerados pela perícia como sem condições de realização normal das atividades, podendo, entretanto, realizar parte das tarefas. Além disso, esse processo colabora para que o trabalhador não se sinta excluído socialmente, sem de utilidade e capacidade, pois se tem a possibilidade de continuar trabalhando (BATISTA; JULIANI; AYRES, 2010).

Diante disso, é relevante realizar a análise da carga de trabalho e da ergonomia destes trabalhadores, associando com o perfil sócio demográfico e ocupacional, para traçar medidas com o objetivo de manutenção e/ou recuperação da capacidade laboral e da qualidade de vida dos servidores.

\section{Conclusões}

Frente à caracterização do perfil sócio-demográfico e ocupacional dos funcionários da prefeitura de um campus universitário público, concluiu-se que a masculinização nesta força de trabalho é evidente e que as atividades desenvolvidas por significativa parcela da população requerem esforço físico, podendo levar a perda precoce de sua capacidade funcional.

Vale ressaltar a expressiva proporção de trabalhadores que ocupam cargos de auxiliar operacional, ou seja, atividades como serviços de manutenção em geral, serviços de zeladoria, serviços de vigilância, serviços de carpintaria e jardinagem entre outros, que são consideradas fatores que podem levá-los a agravos de saúde relacionados às suas tarefas diárias.

Algo evidente é o envelhecimento cronológico da força de trabalho em mais da metade da população estudada, ou seja, $78 \%$, a qual apresenta entre 41 a 60 anos, ocorrendo consequentemente o envelhecimento funcional. Destaca-se que a maior parte destes trabalhadores realiza hora-extra e trabalha em período integral.

Diante do exposto, evidencia-se a necessidade de proporcionar condições que favoreçam a prevenção de doenças relacionadas às atividades laborais por meio de ações de uma equipe multidisciplinar com atividades educativas primárias visando à promoção da saúde e a melhoria da qualidade de vida do servidor que está em envelhecimento cronológico e funcional, garantindo a manutenção e /ou recuperação de sua capacidade funcional.

\section{Referências}

ANDRADE, P. P.; VEIGA, H. M. S. Avaliação dos trabalhadores acerca de um programa de qualidade de vida no trabalho: validação de escala e análise qualitativa. Psicologia: Ciência e Profissão, Brasília, v. 32, n. 2, 2012.

BATISTA, J. M.; JULIANI, C. M. C. M.; AYRES, J. A. O processo de readaptação funcional e suas implicações no gerenciamento em enfermagem. Revista Latino-Americana de Enfermagem, Ribeirão Preto, v. 18, n. 1, p. 87-93, 2010.

BRUSCHINI, M. C. A.; RICOLDI, A. M. Família e trabalho: difícil conciliação para mães trabalhadoras de baixa renda. Cadernos de Pesquisa, São Paulo, v. 39, n. 136, p. 93-123, 2009.

CAMARANO, A. A.; PASINATO, M. T. Envelhecimento funcional e suas implicações para oferta da força de trabalho brasileira. Rio de Janeiro: IPEA, 2008. (Textos para discussão). 
COSTA, D.; LACAZ, F. A. C.; FILHO JACKSON, J. M.; VILELA, R. A. G. Saúde do trabalhador no SUS: desafios para uma política pública. Revista Brasileira de Saúde Ocupacional, São Paulo, v. 38, n. 127, p. 11-30, 2013.

DOMANSKY, R. C. Adaptação transcultural e validação do instrumento "Bowel Function in the Community” para a língua portuguessa. 2004. Dissertação (Mestrado) - Escola de Enfermagem, Universidade de São Paulo, São Paulo, 2004.

FELLI, V. E. A.; TRONCHIN, D. M. R. A qualidade de vida no trabalho e a saúde do trabalhador de enfermagem. In: KURCGANT, P. Gerenciamento em enfermagem. 2. ed. Rio de Janeiro: Guanabara Koogan, 2010. p. 89-107

FISCHER, F. M. Vovô antes da hora. Disponível em: $<$ http://www.usp.br/espacoaberto/arquivo/2006/ espaco67mai/atualiza/comportamento.htm $>$. Acesso em: 15 jun. 2014.

FRANÇA, L. H. F. P.; SOARES, D. H. P. Preparação para a aposentadoria como parte da educação ao longo da vida. Psicologia: Ciência e Profissão, Brasília, v. 29, n. 4, p. 738-751, 2009.

LIMA, F. P. A. Ações coordenadas em saúde do trabalhador: uma proposta de atuação suprainstitucional. Revista Brasileira de Saúde Ocupacional, São Paulo, v. 34, n. 119, p. 67-78, 2009.

MARTINEZ, M. C.; LATORRE, M. R. D. O.; FISCHER, F. M. Capacidade para o trabalho: revisão de literatura. Ciência e Saúde Coletiva, Rio de Janeiro, v. 15, supl. 1, p. 1553-1561, 2010.

NEVES, M. J. A. O; BRANQUINHO, N. C. S. S.; PARANAGUÁ, T. T. B.; BARBOSA, M. A.; SIQUEIRA, K. M. Influência do trabalho noturno na qualidade de vida do enfermeiro. Revista de Enfermagem UERJ, Rio de Janeiro, v. 18, n. 1, p. 42-47, 2010.

PASKULINL, L. M. G.; VIANNALL, L. A. C. Perfil sociodemográfico e condições de saúde auto referidas de idosos de Porto Alegre. Revista de Saúde Pública, São Paulo, v. 41, n. 5, p. 757-68, 2007.
PIRES, A. S.; RIBEIRO, L. V.; SOUZA, N. V. D. O.; SÁ, C. M. S.; GONÇALVES, F. G. Z.; SANTOS, D. M. A permanência no mundo do trabalho de profissionais de enfermagem com possibilidade de aposentadoria. Ciência Cuidado e Saude, Maringá, v. 12, n. 2, p. 338-345, 2013.

QUEMELO, P. R. V; ARAR, M. B.; COELHO, A. R.; GOTTARDO, D. Avaliação ergonômica em empresas calçadistas. Colloquium Vitae, Presidente Prudente, v. 1, n. 2, p. 95-100, 2009.

RIBEIRO, R. P.; MARTINS, J. T.; MARZIALE, M. H. P.; ROBAZZI, M. L. C. C. O adoecer pelo trabalho na enfermagem: uma revisão integrativa. Revista da Escola de Enfermagem da USP, São Paulo, v. 46, n. 2, 2012.

SADIR, M. A.; BIGNOTTO, M. M.; LIPP, M. E. N. Stress e qualidade de vida: influência de algumas variáveis pessoais. Paidéia, Ribeirão Preto, v. 20, n. 45, p. 73-81, 2010.

SANTOS, T. S. Divisão sexual do trabalho na indústria calçadista do Vale do Sinos, Rio Grande do Sul: visibilizando práticas e representações 2004. Mulher e Trabalho, Porto Alegre, v. 6, 2006.

UNIVERSIDADE ESTADUAL DE LONDRINA. Prefeitura Do Campus Universitário - PCU. Disponível em: <http://www.uel.br/pcu/portal/>. Acesso em: 31 jul. 2014a.

Pró-Reitoria de Planejamento - PROPLAN. Dados e números da UEL. Disponível em: $<\mathrm{http}: / /$ www.uel.br/proplan/?content=dadosuel.html $>$. Acesso em: 31 jul. 2014 b. 